\title{
Forced Transverse Vibration of a Closed Double Single-Walled Carbon Nanotube System Containing a Fluid with Effect of Compressive Axial Load
}

\author{
Mehrdad Nasirshoaibi, ${ }^{1}$ Nader Mohammadi, ${ }^{1}$ and Masih Nasirshoaibi ${ }^{2}$ \\ ${ }^{1}$ Department of Mechanical Engineering, Islamic Azad University, Parand Branch, Tehran 3761396361, Iran \\ ${ }^{2}$ Department of Mechanical Engineering, Islamic Azad University, Semnan Branch, Semnan 8965151567, Iran \\ Correspondence should be addressed to Mehrdad Nasirshoaibi; mehrdadnasirshoaibi@gmail.com
}

Received 19 April 2015; Revised 29 July 2015; Accepted 12 August 2015

Academic Editor: Salvatore Strano

Copyright (C) 2015 Mehrdad Nasirshoaibi et al. This is an open access article distributed under the Creative Commons Attribution License, which permits unrestricted use, distribution, and reproduction in any medium, provided the original work is properly cited.

Based on the Rayleigh beam theory, the forced transverse vibrations of a closed double single-walled carbon nanotube (SWCNT) system containing a fluid with a Pasternak layer in-between are investigated. It is assumed that the two single-walled carbon nanotubes of the system are continuously joined by a Pasternak layer and both sides of SWCNTs containing a fluid are closed. The dynamic responses of the system caused by arbitrarily distributed continuous loads are obtained. The effect of compressive axial load on the forced vibrations of the double single-walled carbon nanotube system is discussed for one case of particular excitation loading. The properties of the forced transverse vibrations of the system are found to be significantly dependent on the compressive axial load. The steady-state vibration amplitudes of the SWCNT decrease with increasing of length of SWCNT. Vibrations caused by the harmonic exciting forces are discussed, and conditions of resonance and dynamic vibration absorption are formulated. The SWCNT-type dynamic absorber is a new concept of a dynamic vibration absorber (DVA), which can be applied to suppress excessive vibrations of corresponding SWCNT systems.

\section{Introduction}

Carbon nanotubes (CNTs) are becoming increasingly promising materials for modern technology applications (nanocomposites, nanodevices, nanoelectronics, and nanomedicine) due to their exceptional thermomechanical, chemical, and optoelectronic properties. The superior strength and unusual deformability of CNTs have been a subject of numerous recent studies. For example, the classical Euler and Tim oshenko elastic-beam models have been used effectively to study the deformation of CNTs, including static deflections, column buckling, resonant frequencies, and modes, as well as wave propagation such as Wong et al. [1], Poncharal et al. [2] Yoon et al. [3], Li and Chou [4], Ru [5], Wang and Hu [6], Zhang et al. [7], and Wang et al. [8].

Recently, carbon nanotube (CNT) filled with fluids has been of a great interest. This is because CNTs promise many new applications in nanobiological devices and nanomechanical systems such as fluid conveyance and drug delivery.
Chang and Lee [9] studied the free vibration of a singlewalled carbon nanotube containing a fluid flow by using the Timoshenko beam model. They analyzed the effects of the flow velocity and the aspect ratio of length to diameter on the vibration frequency and mode shape of the SWCNT. Their results showed the effects of rotary inertia and transverse shear deformation result in a reduction of the vibration frequencies, especially for higher modes of vibration and short nanotubes.

The systems connected by an elastic layer constitute one group of such mechanical structures which are commonly encountered in mechanical, construction, and aeronautical industry. Such systems are important as they give the initial approximation of the solution and dynamic behavior of the system at slight motion. The problem of two elastically connected beams joined by the Winkler elastic layer emerged in order to determine the conditions for the behavior of the system acting as a dynamic absorber in technical practice. 
A mathematical model was developed by Seelig and Hoppmann [10]. They investigated the problem of an impulse load effect on a beam and produced a system of partial differential equations describing its vibration. The obtained theoretical and experimental results confirmed a sound approximation of an analytical solution obtained for slender beams at small transverse motions using the Euler-Bernoulli theory.

Oniszczuk $[11,12]$ analyzed the problem of free and forced vibration of two elastically connected Euler-Bernoulli beams. $\mathrm{He}$ determined analytical solutions for eigenfrequencies, amplitudinous functions, and vibration modes. He discussed the effect of stiffness which the elastic interlayer had on the frequencies and amplitudes of the system. Also, the conditions for the occurrence of resonance and the behavior of the system as a dynamic absorber are determined.

The analysis of the system composed of two connected beams was carried on by Zhang et al. [13, 14]. In their work, free and forced vibrations by two elastically connected EulerBernoulli beams affected by axial compression forces are investigated. They presented analytical solutions for natural frequencies of the system in the function of axial compression force impact and their effect on the vibration amplitude. They determined the codependency between the system's critical force and the Euler critical load in the function of an axial force of the other beam.

Stojanović et al. [15] analyzed free vibration and static stability of two elastically connected beams with Winkler elastic layer in-between with the influence of rotary inertia and transverse shear. They described the motion of the system by a homogeneous set of two partial differential equations. Also, they determined the natural frequencies and associated amplitude ratios of an elastically connected double-beam complex system and the analytical solution of the critical buckling load.

Stojanović and Kozić [16] discussed the case of forced vibration of two elastically connected beams with Winkler elastic layer in-between and the effect of axial compression force on the amplitude ratio of system vibration for three types of external forcing (arbitrarily continuous harmonic excitation, uniformly continuous harmonic excitation, and concentrated harmonic excitation). They determined general conditions of resonance and dynamic vibration absorption. In the other paper, Stojanović et al. [17] discussed the analytic analysis of static stability of a system consisting of three elastically connected Timoshenko beams on an elastic foundation. They provided expressions for critical force of the system under the influence of elastic Winkler layers.

In an attempt to find a physically close and mathematically simple representation of an elastic foundation for these materials, Pasternak proposed a foundation model consisting of a Winkler foundation with shear interactions. This may be accomplished by connecting the ends of the vertical springs to a beam consisting of incompressible vertical elements, which deforms only by transverse shear.

\section{The Beam Model for SWCNTs}

The Rayleigh beam theory, which has taken rotary inertia into account, is applied to analyze forced transverse vibration of

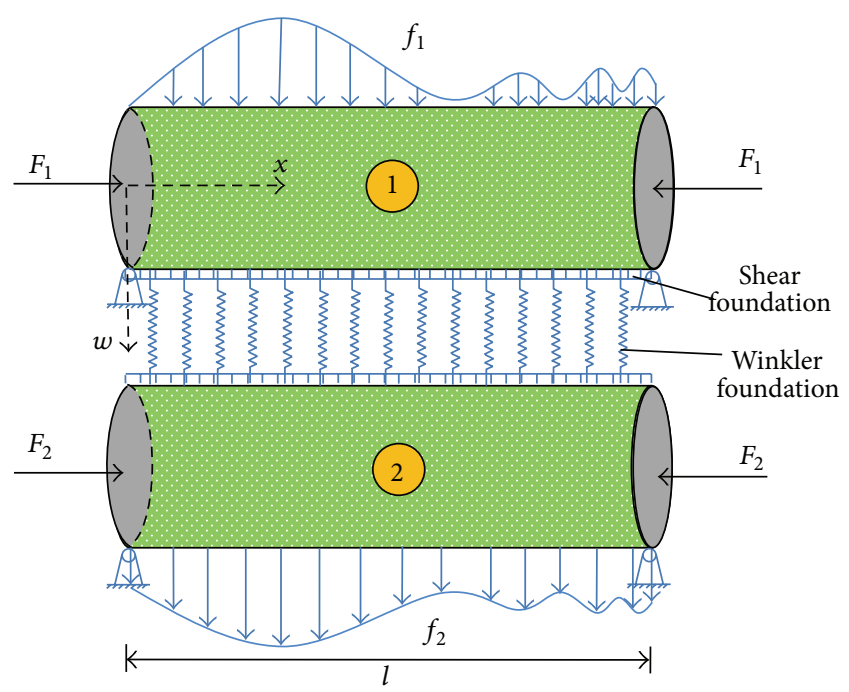

FIGURE 1: Double single-walled carbon nanotube system.

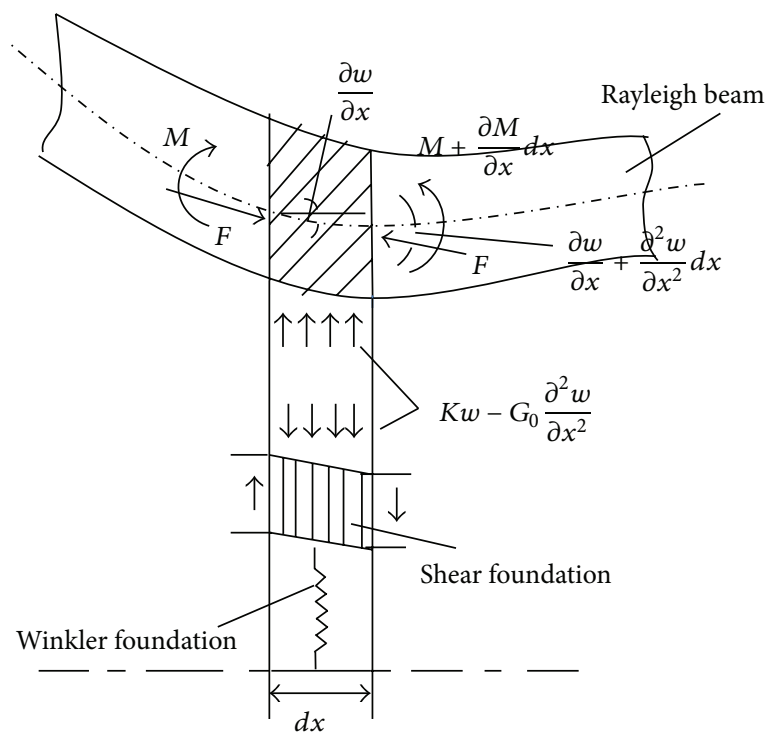

Figure 2: Deflected differential Rayleigh beam element with Pasternak layer in-between.

a closed double SWCNT system containing a fluid with effect of compressive axial load with Pasternak interlayer.

Figure 1 shows a double SWCNT system containing a fluid with a Pasternak layer in-between with length of $l$, which was subjected to axial compressions $F_{1}$ and $F_{2}$ that are positive in compression, which do not change with time and arbitrarily distributed transverse continuous loads $f_{1}$ and $f_{2}$ that are positive when they act downward.

An element of deflected differential Rayleigh beam of length $d x$ with Pasternak layer between two cross sections is shown in Figure 2. 
Let the functions of longitudinal and transverse motion of the Rayleigh beam system be as follows: $u_{R 1}(y, x, t)$, $w_{R 1}(y, x, t), u_{R 2}(y, x, t)$, and $w_{R 2}(y, x, t)$. We have

$$
\begin{aligned}
& u_{R 1}(y, x, t)=u_{R 1}^{0}(x, t)-y \frac{\partial w_{R 1}^{0}(x, t)}{\partial x}, \\
& w_{R 1}(y, x, t)=w_{R 1}^{0}(x, t), \\
& u_{R 2}(y, x, t)=u_{R 2}^{0}(x, t)-y \frac{\partial w_{R 2}^{0}(x, t)}{\partial x}, \\
& w_{R 2}(y, x, t)=w_{R 2}^{0}(x, t) .
\end{aligned}
$$

Deformation in the $x$ direction in the function of motion and the stress-deformation relation according to Hooke's law are as follows:

$$
\begin{aligned}
\varepsilon_{x 1}(y, x, t) & =\frac{\partial u_{R 1}^{0}(x, t)}{\partial z}-y \frac{\partial^{2} w_{R 1}^{0}(x, t)}{\partial z^{2}}, \\
\varepsilon_{x 2}(y, x, t) & =\frac{\partial u_{R 2}^{0}(x, t)}{\partial z}-y \frac{\partial^{2} w_{R 2}^{0}(x, t)}{\partial z^{2}}, \\
\sigma_{x 1}(y, x, t) & =E \varepsilon_{x 1}(y, x, t) \\
\sigma_{x 2}(y, x, t) & =E \varepsilon_{x 2}(y, x, t) .
\end{aligned}
$$

Virtual work of inertial forces is expressed as

$$
\begin{gathered}
\delta W_{i n 1}=-\left(\rho_{C 1}+\rho_{f 1}\right) \\
\cdot b_{1} \int_{0}^{l} \int_{-h_{1} / 2}^{h_{1} / 2}\left[\frac{\partial^{2} w_{R 1}(y, x, t)}{\partial t^{2}} \delta w_{R 1}(y, x, t)\right. \\
\left.+\frac{\partial^{2} u_{R 1}(y, x, t)}{\partial t^{2}} \delta u_{R 1}(y, x, t)\right] d y d x, \\
\delta W_{i n 2}=-\left(\rho_{C 2}+\rho_{f 2}\right) \\
\cdot b_{2} \int_{0}^{l} \int_{-h_{2} / 2}^{h_{2} / 2}\left[\frac{\partial^{2} w_{R 2}(y, x, t)}{\partial t^{2}} \delta w_{R 2}(y, x, t)\right. \\
\left.+\frac{\partial^{2} u_{R 2}(y, x, t)}{\partial t^{2}} \delta u_{R 2}(y, x, t)\right] d y d x .
\end{gathered}
$$

Virtual work of internal forces is

$$
\begin{aligned}
& \delta W_{V 1}=-b_{1} \int_{0}^{l} \int_{-h_{1} / 2}^{h_{1} / 2} \sigma_{x 1}(x, t) \delta \varepsilon_{x 1}(x, t) d y d x, \\
& \delta W_{V 2}=-b_{2} \int_{0}^{l} \int_{-h_{2} / 2}^{h_{2} / 2} \sigma_{x 2}(x, t) \delta \varepsilon_{x 2}(x, t) d y d x .
\end{aligned}
$$

Virtual work of external forces is given by

$$
\begin{gathered}
\delta W_{e x 1}=\int_{0}^{l}\left[\delta w_{R 1}^{0}(x, t) K\left(w_{R 2}^{0}(x, t)-w_{R 1}^{0}(x, t)\right)\right. \\
-G_{0}\left(\frac{\partial w_{R 2}^{0}(x, t)}{\partial x} \frac{\partial \delta w_{R 2}^{0}(x, t)}{\partial x}\right. \\
\left.-\frac{\partial w_{R 1}^{0}(x, t)}{\partial x} \frac{\partial \delta w_{R 1}^{0}(x, t)}{\partial x}\right)+F_{1} \frac{\partial w_{R 1}^{0}(x, t)}{\partial x} \\
\left.. \frac{\partial \delta w_{R 1}^{0}(x, t)}{\partial x}\right] d x, \\
\delta W_{e x 2}=\int_{0}^{l}\left[\delta w_{R 2}^{0}(x, t) K\left(w_{R 1}^{0}(x, t)-w_{R 2}^{0}(x, t)\right)\right. \\
-G_{0}\left(\frac{\partial w_{R 1}^{0}(x, t)}{\partial x} \frac{\partial \delta w_{R 1}^{0}(x, t)}{\partial x}\right. \\
\left.-\frac{\partial w_{R 2}^{0}(x, t)}{\partial x} \frac{\partial \delta w_{R 2}^{0}(x, t)}{\partial x}\right)+F_{2} \frac{\partial w_{R 2}^{0}(x, t)}{\partial x} \\
\left.. \frac{\partial \delta w_{R 2}^{0}(x, t)}{\partial x}\right] d x .
\end{gathered}
$$

Based on the principle of virtual work $\delta W_{i n i}+\delta W_{V i}+\delta W_{\text {exi }}=$ $0, i=1,2$, and (3) to (5), we have

$$
\begin{aligned}
& -b_{1} \int_{0}^{l} \int_{-h_{1} / 2}^{h_{1} / 2} \sigma_{x 1}(x, t) \delta \varepsilon_{x 1}(x, t) d y d x-\left(\rho_{C 1}+\rho_{f 1}\right) \\
& \cdot b_{1} \int_{0}^{l} \int_{-h_{1} / 2}^{h_{1} / 2}\left[\frac{\partial^{2} w_{R 1}(y, x, t)}{\partial t^{2}} \delta w_{R 1}(y, x, t)\right. \\
& \left.+\frac{\partial^{2} u_{R 1}(y, x, t)}{\partial t^{2}} \delta u_{R 1}(y, x, t)\right] d y d x \\
& +\int_{0}^{l}\left[\delta w_{R 1}^{0}(x, t) K\left(w_{R 2}^{0}(x, t)-w_{R 1}^{0}(x, t)\right)\right. \\
& -G_{0}\left(\frac{\partial w_{R 2}^{0}(x, t)}{\partial x} \frac{\partial \delta w_{R 2}^{0}(x, t)}{\partial x}\right. \\
& \left.-\frac{\partial w_{R 1}^{0}(x, t)}{\partial x} \frac{\partial \delta w_{R 1}^{0}(x, t)}{\partial x}\right)+F_{1} \frac{\partial w_{R 1}^{0}(x, t)}{\partial x} \\
& \left.\quad \cdot \frac{\partial \delta w_{R 1}^{0}(x, t)}{\partial x}\right] d x=0, \\
& -b_{2} \int_{0}^{l} \int_{-h_{2} / 2}^{h_{2} / 2} \sigma_{x 2}(x, t) \delta \varepsilon_{x 2}(x, t) d y d x-\left(\rho_{C 2}+\rho_{f 2}\right) \\
& \cdot b_{2} \int_{0}^{l} \int_{-h_{2} / 2}^{h_{2} / 2}\left[\frac{\partial^{2} w_{R 2}(y, x, t)}{\partial t^{2}} \delta w_{R 2}(y, x, t)\right. \\
& \left.+\frac{\partial^{2} u_{R 2}(y, x, t)}{\partial t^{2}} \delta u_{R 2}(y, x, t)\right] d y d x
\end{aligned}
$$




$$
\begin{aligned}
& +\int_{0}^{l}\left[\delta w_{R 2}^{0}(x, t) K\left(w_{R 1}^{0}(x, t)-w_{R 2}^{0}(x, t)\right)\right. \\
& -G_{0}\left(\frac{\partial w_{R 1}^{0}(x, t)}{\partial x} \frac{\partial \delta w_{R 1}^{0}(x, t)}{\partial x}\right. \\
& \left.-\frac{\partial w_{R 2}^{0}(x, t)}{\partial x} \frac{\partial \delta w_{R 2}^{0}(x, t)}{\partial x}\right)+F_{2} \frac{\partial w_{R 2}^{0}(x, t)}{\partial x} \\
& \left.\cdot \frac{\partial \delta w_{R 2}^{0}(x, t)}{\partial x}\right] d x=0 .
\end{aligned}
$$

By successive application of Green's theorem on expressions (6), we obtain the set of coupled differential equations of forced transverse vibration of a double SWCNT system containing a fluid with effect of compressive axial load with Pasternak layer in-between:

$$
\begin{aligned}
& E I_{1} \frac{\partial^{4} w_{1}}{\partial x^{4}}+\left(\rho_{C 1}+\rho_{f 1}\right) A_{1} \frac{\partial^{2} w_{1}}{\partial t^{2}} \\
& -\left(\rho_{C 1}+\rho_{f 1}\right) I_{1} \frac{\partial^{4} w_{1}}{\partial x^{2} \partial t^{2}}+F_{1} \frac{\partial^{2} w_{1}}{\partial x^{2}}+K\left(w_{1}-w_{2}\right) \\
& -G_{0}\left(\frac{\partial^{2} w_{1}}{\partial x^{2}}-\frac{\partial^{2} w_{2}}{\partial x^{2}}\right)=f_{1}(x, t), \\
& E I_{2} \frac{\partial^{4} w_{2}}{\partial x^{4}}+\left(\rho_{C 2}+\rho_{f 2}\right) A_{2} \frac{\partial^{2} w_{1}}{\partial t^{2}} \\
& -\left(\rho_{C 2}+\rho_{f 2}\right) I_{2} \frac{\partial^{4} w_{2}}{\partial x^{2} \partial t^{2}}+F_{2} \frac{\partial^{2} w_{2}}{\partial x^{2}}+K\left(w_{2}-w_{1}\right) \\
& -G_{0}\left(\frac{\partial^{2} w_{2}}{\partial x^{2}}-\frac{\partial^{2} w_{1}}{\partial x^{2}}\right)=f_{2}(x, t),
\end{aligned}
$$

where

$$
\begin{aligned}
A_{i} & =\pi d t_{C}, \\
I_{i} & =\frac{\pi d^{3} t_{C}}{8},
\end{aligned}
$$

$$
i=1,2 \text {. }
$$

If we introduce $G_{0}=0$ in the equations, the effect of shear foundation will be omitted and we can obtain the vibration equations of a double SWCNT system containing a fluid with Winkler elastic layer in-between.

Equations (7) can be reduced to fourth-order partial differential equations for forced vibration of a double SWCNT system model:

$$
\begin{gathered}
C_{b 1}^{2} \frac{\partial^{4} w_{1}}{\partial x^{4}}+\frac{\partial^{2} w_{1}}{\partial t^{2}}-C_{r 1}^{2} \frac{\partial^{4} w_{1}}{\partial x^{2} \partial t^{2}}+m_{1}\left(F_{1}-G_{0}\right) \frac{\partial^{2} w_{1}}{\partial x^{2}} \\
+m_{1} G_{0} \frac{\partial^{2} w_{2}}{\partial x^{2}}+H_{1}\left(w_{1}-w_{2}\right)=m_{1} f_{1}(x, t)
\end{gathered}
$$

$$
\begin{gathered}
C_{b 2}^{2} \frac{\partial^{4} w_{2}}{\partial x^{4}}+\frac{\partial^{2} w_{2}}{\partial t^{2}}-C_{r 2}^{2} \frac{\partial^{4} w_{2}}{\partial x^{2} \partial t^{2}}+m_{2}\left(F_{2}-G_{0}\right) \frac{\partial^{2} w_{2}}{\partial x^{2}} \\
+m_{2} G_{0} \frac{\partial^{2} w_{1}}{\partial x^{2}}+H_{2}\left(w_{2}-w_{1}\right)=m_{2} f_{2}(x, t),
\end{gathered}
$$

where

$$
\begin{aligned}
m_{i} & =\frac{1}{\left(\rho_{C i}+\rho_{f i}\right) A_{i}}, \\
H_{i} & =\frac{K}{\left(\rho_{C i}+\rho_{f i}\right) A_{i}}, \\
C_{b i} & =\sqrt{\frac{E I_{i}}{\left(\rho_{C i}+\rho_{f i}\right) A_{i}}}, \\
C_{r i} & =\sqrt{\frac{I_{i}}{A_{i}},} \quad i=1,2 .
\end{aligned}
$$

The initial conditions in general form and boundary conditions for simply supported SWCNT of the same length $l$ are assumed as follows:

$$
\begin{array}{ll}
w_{i}(x, 0) & =w_{i 0}(x), \\
\dot{w}_{i}(x, 0) & =v_{i 0}(x), \\
w_{i}(0, t)=w_{i}^{\prime \prime}(0, t)=w_{i}(l, t)=w_{i}^{\prime \prime}(l, t)=0, & \\
i=1,2 .
\end{array}
$$

\section{Solution of Equations}

Equations (9) and (10) represent forced vibrations of a double SWCNT system. The natural frequencies and the corresponding mode shapes of the system should be obtained by solving the undamped free vibration with appropriate boundary conditions. Assuming time harmonic motion and using separation of variables, the solutions to (9) and (10) with the governing boundary conditions (13) can be written in the form

$$
w_{i}(x, t)=\sum_{n=1}^{\infty} X_{n}(x) T_{n i}(t)
$$

where

$$
\begin{aligned}
X_{n}(x) & =\sin \left(k_{n} x\right), \\
k_{n} & =\frac{n \pi}{l},
\end{aligned}
$$

$$
n=1,2,3, \ldots
$$


Substitution of (14) into (9) and (10) yields ordinary differential equations for the double SWCNT system

$$
\begin{gathered}
\sum_{n=1}^{\infty}\left[J_{1} \frac{d^{2} T_{n 1}}{d t^{2}}+\left(N_{1}-F_{1} \eta_{1}+G_{0} \eta_{1}\right) T_{n 1}\right. \\
\left.-\left(H_{1}+G_{0} \eta_{1}\right) T_{n 2}\right] X_{n}=m_{1} f_{1}, \\
\sum_{n=1}^{\infty}\left[J_{2} \frac{d^{2} T_{n 2}}{d t^{2}}+\left(N_{2}-F_{2} \eta_{2}+G_{0} \eta_{2}\right) T_{n 2}\right. \\
\left.-\left(H_{2}+G_{0} \eta_{2}\right) T_{n 1}\right] X_{n}=m_{2} f_{2},
\end{gathered}
$$

where

$$
\begin{aligned}
& \eta_{1}=\frac{k_{n}^{2}}{\rho A_{1}}, \\
& \eta_{2}=\frac{k_{n}^{2}}{\rho A_{2}}, \\
& J_{1}=1+C_{r 1}^{2} k_{n}^{2}, \\
& J_{2}=1+C_{r 2}^{2} k_{n}^{2}, \\
& N_{1}=C_{b 1}^{2} k_{n}^{4}+H_{1}, \\
& N_{2}=C_{b 2}^{2} k_{n}^{4}+H_{2} .
\end{aligned}
$$

\section{Forced Vibration of a Double SWCNT System}

First, we should solve the undamped free vibration of the double SWCNT system

$$
\begin{aligned}
& J_{1} \frac{d^{2} T_{n 1}}{d t^{2}}+\left(N_{1}-F_{1} \eta_{1}+G_{0} \eta_{1}\right) T_{n 1}-\left(H_{1}+G_{0} \eta_{1}\right) T_{n 2} \\
& \quad=0 \\
& J_{2} \frac{d^{2} T_{n 1}}{d t^{2}}+\left(N_{2}-F_{2} \eta_{2}+G_{0} \eta_{2}\right) T_{n 2}-\left(H_{2}+G_{0} \eta_{2}\right) T_{n 1} \\
& \quad=0 .
\end{aligned}
$$

The solutions of (18) can be assumed to have the following forms:

$$
\begin{aligned}
& T_{n 1}=C_{n} e^{i \omega_{n} t}, \\
& T_{n 2}=D_{n} e^{i \omega_{n} t} .
\end{aligned}
$$

Substituting (19) into (18) results in the following system of homogeneous algebraic equations for the unknown constants:

$$
\begin{aligned}
& \left(N_{1}-F_{1} \eta_{1}+G_{0} \eta_{1}-J_{1} \omega_{n}^{2}\right) C_{n}-\left(H_{1}+G_{0} \eta_{1}\right) D_{n} \\
& \quad=0 \\
& -\left(H_{2}+G_{0} \eta_{2}\right) C_{n}+\left(N_{2}-F_{2} \eta_{2}+G_{0} \eta_{2}-J_{2} \omega_{n}^{2}\right) D_{n} \\
& \quad=0 .
\end{aligned}
$$

When the determinant of the coefficients in (20) vanishes, nontrivial solutions for the constants $C_{n}$ and $D_{n}$ can be obtained, which yield the following frequency (characteristic) equation:

$$
\begin{aligned}
& J_{1} J_{2} \omega_{n}^{4}-\left(N_{1} J_{2}+N_{2} J_{1}-F_{1} \eta_{1} J_{2}-F_{2} \eta_{2} J_{1}+G_{0} \eta_{1} J_{2}\right. \\
& \left.+G_{0} \eta_{2} J_{1}\right) \omega_{n}^{2}+\left(N_{1}-F_{1} \eta_{1}\right)\left(N_{2}-F_{2} \eta_{2}\right)-H_{1} H_{2} \\
& \quad-G_{0}\left(H_{1} \eta_{2}+H_{2} \eta_{1}-N_{1} \eta_{2}-N_{2} \eta_{1}+F_{1} \eta_{1} \eta_{2}\right. \\
& \left.\quad+F_{2} \eta_{1} \eta_{2}\right)=0 .
\end{aligned}
$$

The roots of (21) are

$$
\begin{aligned}
& \omega_{n I}^{2} \\
& =\frac{J_{2}\left(N_{1}-F_{1} \eta_{1}\right)+J_{1}\left(N_{2}-F_{2} \eta_{2}\right)+G_{0}\left(\eta_{1} J_{2}+\eta_{2} J_{1}\right)}{2 J_{1} J_{2}} \\
& \quad-\frac{1}{2 J_{1} J_{2}} \sqrt{D}, \\
& \omega_{n I I}^{2} \\
& =\frac{J_{2}\left(N_{1}-F_{1} \eta_{1}\right)+J_{1}\left(N_{2}-F_{2} \eta_{2}\right)+G_{0}\left(\eta_{1} J_{2}+\eta_{2} J_{1}\right)}{2 J_{1} J_{2}} \\
& \quad+\frac{1}{2 J_{1} J_{2}} \sqrt{D},
\end{aligned}
$$

where

$$
\begin{aligned}
D & =\left[J_{2}\left(N_{1}-F_{1} \eta_{1}\right)-J_{1}\left(N_{2}-F_{2} \eta_{2}\right)\right]^{2}+4 J_{1} J_{2} H_{1} H_{2} \\
& +G_{0}^{2}\left(\eta_{1} J_{2}+\eta_{2} J_{1}\right)^{2}+2 J_{2}^{2} G_{0} \eta_{1}\left(N_{1}-F_{1} \eta_{1}\right) \\
& +2 J_{1}^{2} G_{0} \eta_{2}\left(N_{2}-F_{2} \eta_{2}\right)+2 J_{1} J_{2} G_{0}\left(2 H_{1} \eta_{2}+2 H_{2} \eta_{1}\right. \\
& \left.-N_{1} \eta_{2}-N_{2} \eta_{1}+F_{1} \eta_{1} \eta_{2}+F_{2} \eta_{1} \eta_{2}\right) .
\end{aligned}
$$

For each of the natural frequencies, the associated amplitude ratio of vibration modes of the two beams is given by

$$
\begin{aligned}
\alpha_{n i}^{-1} & =\frac{C_{n}}{D_{n}}=\frac{H_{1}+G_{0} \eta_{1}}{N_{1}-F_{1} \eta_{1}+G_{0} \eta_{1}-J_{1} \omega_{n i}^{2}} \\
& =\frac{N_{2}-F_{2} \eta_{2}+G_{0} \eta_{2}-J_{2} \omega_{n i}^{2}}{H_{2}+G_{0} \eta_{2}}, \quad i=I, I I .
\end{aligned}
$$


Applying the classical modal expansion method, the forced vibrations of a double SWCNT system subjected to arbitrarily distributed continuous loads can be determined. Following the above analysis for the undamped free transverse vibration, particular solutions of nonhomogeneous differential equations (9) and (10) representing forced vibrations of a double SWCNT system model can be assumed in the following:

$$
\begin{aligned}
& w_{1}(x, t)=\sum_{n=1}^{\infty}\left[X_{n}(x) \sum_{i=I}^{I I} S_{n i}(t)\right], \\
& w_{2}(x, t)=\sum_{n=1}^{\infty}\left[X_{n}(x) \sum_{i=I}^{I I} \alpha_{n i} S_{n i}(t)\right] .
\end{aligned}
$$

Introduction of (25) and (26) into (9) and (10) results in

$$
\begin{aligned}
& \sum_{n=1}^{\infty}\left\{X_{n}(x)\right. \\
& \left.\quad \sum_{i=I}^{I I}\left[J_{1} \ddot{S}_{n i}+\left(N_{1}-\eta_{1} F_{1}+G_{0} \eta_{1}-\eta_{1} G_{0} \alpha_{n i}-H_{1} \alpha_{n i}\right) S_{n i}\right]\right\} \\
& =m_{1} f_{1}, \\
& \sum_{n=1}^{\infty}\left\{X_{n}(x)\right. \\
& \cdot \sum_{i=I}^{I I}\left[J_{2} \ddot{S}_{n i}+\left(N_{2}-\eta_{2} F_{2}+G_{0} \eta_{2}-\eta_{2} G_{0} \alpha_{n i}^{-1}-H_{2} \alpha_{n i}^{-1}\right) S_{n i}\right] \\
& \left.\quad \cdot \alpha_{n i}\right\}=m_{2} f_{2} .
\end{aligned}
$$

By multiplying relations (27) and (28) by the eigenfunction $X_{m}$ and then integrating them with respect to $x$ from 0 to $l$ and using orthogonality condition

$$
\int_{0}^{l} X_{m} X_{n} d x=\int_{0}^{l} \sin \left(k_{n} x\right) \sin \left(k_{m} x\right) d x=c \delta_{m n},
$$

where

$$
c=\int_{0}^{l} X_{n}^{2}=\int_{0}^{l}\left[\sin \left(k_{n} x\right)\right]^{2} d x=\frac{l}{2},
$$

now we have

$$
\begin{aligned}
\sum_{i=I}^{I I} & {\left[J_{1} \ddot{S}_{n i}\right.} \\
& \left.+\left(N_{1}-\eta_{1} F_{1}+G_{0} \eta_{1}-\eta_{1} G_{0} \alpha_{n i}-H_{1} \alpha_{n i}\right) S_{n i}\right] \\
& =\frac{2 m_{1}}{l} \int_{0}^{l} X_{n} f_{1} d x,
\end{aligned}
$$

$$
\begin{aligned}
\sum_{i=I}^{I I} & {\left[J_{2} \ddot{S}_{n i}\right.} \\
& \left.+\left(N_{2}-\eta_{2} F_{2}+G_{0} \eta_{2}-\eta_{2} G_{0} \alpha_{n i}^{-1}-H_{2} \alpha_{n i}^{-1}\right) S_{n i}\right] \alpha_{n i} \\
& =\frac{2 m_{2}}{l} \int_{0}^{l} X_{n} f_{2} d x .
\end{aligned}
$$

From (24) and (31) after some algebra we obtain

$$
\begin{aligned}
\sum_{i=I}^{I I}\left(\ddot{S}_{n i}+\omega_{n i}^{2} S_{n i}\right) & =\frac{2 m_{1}}{J_{1} l} \int_{0}^{l} X_{n} f_{1} d x, \\
\sum_{i=I}^{I I}\left(\ddot{S}_{n i}+\omega_{n i}^{2} S_{n i}\right) \alpha_{n i} & =\frac{2 m_{2}}{J_{2} l} \int_{0}^{l} X_{n} f_{2} d x .
\end{aligned}
$$

From (32), we obtain

$$
\ddot{S}_{n i}+\omega_{n i}^{2} S_{n i}=Z_{n i}(t), \quad i=I, I I,
$$

where

$$
\begin{aligned}
& Z_{n I}(t) \\
& \quad=\frac{2}{\alpha_{n I I}-\alpha_{n I}} \int_{0}^{l}\left(M_{1} \alpha_{n I I} f_{1}-M_{2} f_{2}\right) \sin \left(k_{n} x\right) d x, \\
& Z_{n I I}(t) \\
& \quad=\frac{2}{\alpha_{n I}-\alpha_{n I I}} \int_{0}^{l}\left(M_{1} \alpha_{n I} f_{1}-M_{2} f_{2}\right) \sin \left(k_{n} x\right) d x .
\end{aligned}
$$

Also, $M_{1}=m_{1} / J_{1} l$ and $M_{2}=m_{2} / J_{2} l$. By combining (25), (26), and (33), the forced vibrations of a double SWCNT system with Pasternak layer in-between which contains a fluid can be described by

$$
\begin{array}{r}
w_{1}(x, t)=\sum_{n=1}^{\infty}\left\{\sin \left(k_{n} x\right)\right. \\
\left.\cdot \sum_{i=I}^{I I} \frac{1}{\omega_{n i}} \int_{0}^{t} Z_{n i}(s) \sin \left[\omega_{n i}(t-s)\right] d s\right\}, \\
w_{2}(x, t)=\sum_{n=1}^{\infty}\left\{\sin \left(k_{n} x\right)\right. \\
\left.\cdot \sum_{i=I}^{I I} \frac{\alpha_{n i}}{\omega_{n i}} \int_{0}^{t} Z_{n i}(s) \sin \left[\omega_{n i}(t-s)\right] d s\right\} .
\end{array}
$$

Now these general solutions (35) and (36) are used to find the vibrations of a double SWCNT system containing a fluid.

In the following, we conduct an analysis of forced vibrations for the case of uniformly distributed continuous harmonic load. For simplicity of further analysis, it is assumed that only one of the two carbon nanotubes is subjected to the exciting load. Without loss of generality, we suppose (see Figure 3)

$$
\begin{aligned}
& f_{1}(x, t)=q \sin (\Omega t), \\
& f_{2}(x, t)=0 .
\end{aligned}
$$




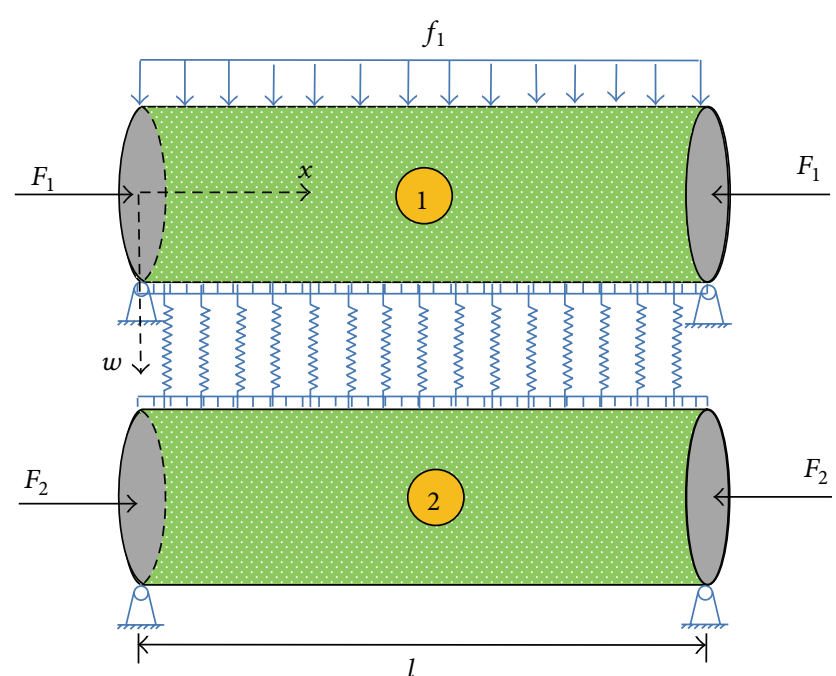

FIGURE 3: Double single-walled carbon nanotube system subjected to harmonic uniform distributed continuous load.

Substituting (37) into (34), we obtain

$$
\begin{aligned}
& Z_{n I}(t)=\frac{\alpha_{n I I}}{\alpha_{n I I}-\alpha_{n I}} M_{R n} \sin (\Omega t), \quad n=1,3,5, \ldots, \\
& Z_{n I I}(t)=\frac{\alpha_{n I}}{\alpha_{n I}-\alpha_{n I I}} M_{R n} \sin (\Omega t), \quad n=1,3,5, \ldots,
\end{aligned}
$$

where

$$
M_{R n}=M_{1} \frac{4 l q}{n \pi}, \quad n=1,3,5, \ldots
$$

Substituting (38) and (39) into (35) and (36) gives

$$
\begin{aligned}
& w_{1}(x, t) \\
& =\sum_{n=1}^{\infty} \sin \left(k_{n} x\right)\left[A_{n 1} \sin (\Omega t)+\sum_{i=I}^{I I} B_{n i} \sin \left(\omega_{n} t\right)\right], \\
& n=1,3,5, \ldots, \\
& w_{2}(x, t) \\
& =\sum_{n=1}^{\infty} \sin \left(k_{n} x\right)\left[A_{n 2} \sin (\Omega t)+\sum_{i=I}^{I I} \alpha_{n i} B_{n i} \sin \left(\omega_{n} t\right)\right], \\
& n=1,3,5, \ldots,
\end{aligned}
$$

where

$$
\begin{aligned}
& A_{n 1}=\frac{M_{R n}}{\alpha_{n I I}-\alpha_{n I}}\left(\frac{\alpha_{n I I}}{\omega_{n I}^{2}-\Omega^{2}}-\frac{\alpha_{n I}}{\omega_{n I I}^{2}-\Omega^{2}}\right), \\
& A_{n 2}=\frac{M_{R n}}{\alpha_{n I I}-\alpha_{n I}}\left(\frac{\alpha_{n I} \alpha_{n I I}}{\omega_{n I}^{2}-\Omega^{2}}-\frac{\alpha_{n I} \alpha_{n I I}}{\omega_{n I I}^{2}-\Omega^{2}}\right),
\end{aligned}
$$

$$
\begin{aligned}
& B_{n I}=M_{R n} \frac{\alpha_{n I I}}{\alpha_{n I I}-\alpha_{n I}}\left[\frac{\Omega}{\omega_{n I}\left(\Omega^{2}-\omega_{n I}^{2}\right)}\right], \\
& B_{n I I}=M_{R n} \frac{\alpha_{n I}}{\alpha_{n I I}-\alpha_{n I}}\left[\frac{\Omega}{\omega_{n I I}\left(\omega_{n I I}^{2}-\Omega^{2}\right)}\right] .
\end{aligned}
$$

Ignoring the free response, the forced vibrations of the double SWCNT system can be obtained by

$$
\begin{aligned}
& w_{1}(x, t)=\sin (\Omega t) \sum_{n=1}^{\infty} A_{n 1} \sin \left(k_{n} x\right), \\
& \quad n=1,3,5, \ldots, \\
& w_{2}(x, t)=\sin (\Omega t) \sum_{n=1}^{\infty} A_{n 2} \sin \left(k_{n} x\right), \\
& \quad n=1,3,5, \ldots .
\end{aligned}
$$

The following fundamental conditions of resonance and dynamic vibration absorption have practical significance:

(1) Resonance

$$
\Omega=\omega_{n i}, \quad n=1,3,5, \ldots
$$

(2) Dynamic vibration absorption

$$
\begin{aligned}
& A_{n 1}=0 \\
& \Omega^{2}=\frac{\alpha_{n I I} \omega_{n I I}^{2}-\alpha_{n I} \omega_{n I}^{2}}{\alpha_{n I I}-\alpha_{n I}}, \\
& A_{n 2}=M_{R n} \frac{\alpha_{n I}-\alpha_{n I I}}{\omega_{n I}^{2}-\omega_{n I I}^{2}} .
\end{aligned}
$$

\section{Numerical Results and Discussion}

For simplicity, it is assumed that both SWCNTs are geometrically and physically identical and the elastic interlayer is Winkler. We assumed that the fluid in the SWCNT is water. The values of the parameters characterizing properties of the system are shown in Table 1:

$$
\begin{aligned}
& H_{1}=H_{2}, \\
& \eta_{1}=\eta_{2}, \\
& F_{2}=\zeta F_{1}, \quad 0 \leq \zeta \leq 1 .
\end{aligned}
$$

If the axial compressions vanish, for the double SWCNT system we have

$$
\begin{aligned}
\left(\omega_{n I}^{0}\right)^{2}= & \frac{J_{2} N_{1}+J_{1} N_{2}+G_{0}\left(\eta_{1} J_{2}+\eta_{2} J_{1}\right)}{2 J_{1} J_{2}} \\
& -\frac{1}{2 J_{1} J_{2}} \sqrt{D^{\prime}}, \\
\left(\omega_{n I I}^{0}\right)^{2}= & \frac{J_{2} N_{1}+J_{1} N_{2}+G_{0}\left(\eta_{1} J_{2}+\eta_{2} J_{1}\right)}{2 J_{1} J_{2}} \\
& +\frac{1}{2 J_{1} J_{2}} \sqrt{D^{\prime}},
\end{aligned}
$$


TABLE 1: Values of the parameters characterizing properties of the system.

\begin{tabular}{lcccc}
\hline$l$ & $E$ & $A$ & $K$ & $G_{0}$ \\
\hline $13.56 \mathrm{~nm}$ & $5.5 \mathrm{TNm}^{-2}$ & $0.140 \mathrm{n}^{2} \mathrm{~m}^{2}$ & $2 \times 10^{5} \mathrm{Nm}^{-2}$ & 0 \\
\hline$\rho_{c}$ & $I$ & $d$ & $t_{C}$ & $\rho_{f}$ \\
\hline $2300 \mathrm{kgm}^{-3}$ & $8.077 \times 10^{-3} \mathrm{n}^{4} \mathrm{~m}^{4}$ & $0.678 \mathrm{~nm}$ & $0.066 \mathrm{~nm}$ & $1000 \mathrm{kgm}^{-3}$ \\
\hline
\end{tabular}

where

$$
\begin{aligned}
D^{\prime}= & {\left[J_{2} N_{1}-J_{1} N_{2}\right]^{2}+4 J_{1} J_{2} H_{1} H_{2} } \\
& +G_{0}^{2}\left(\eta_{1} J_{2}+\eta_{2} J_{1}\right)^{2}+2 J_{2}^{2} G_{0} \eta_{1} N_{1}+2 J_{1}^{2} G_{0} \eta_{2} N_{2} \\
& +2 J_{1} J_{2} G_{0}\left(2 H_{1} \eta_{2}+2 H_{2} \eta_{1}-N_{1} \eta_{2}-N_{2} \eta_{1}\right) .
\end{aligned}
$$

Also,

$$
\begin{aligned}
\left(\alpha_{n I}^{0}\right)^{-1} & =\frac{H_{1}+G_{0} \eta_{1}}{N_{1}+G_{0} \eta_{1}-J_{1}\left(\omega_{n I}^{0}\right)^{2}} \\
& =\frac{N_{2}+G_{0} \eta_{2}-J_{2}\left(\omega_{n I}^{0}\right)^{2}}{H_{2}+G_{0} \eta_{2}}, \\
\left(\alpha_{n I I}^{0}\right)^{-1} & =\frac{H_{1}+G_{0} \eta_{1}}{N_{1}+G_{0} \eta_{1}-J_{1}\left(\omega_{n I}^{0}\right)^{2}} \\
& =\frac{N_{2}+G_{0} \eta_{2}-J_{2}\left(\omega_{n I I}^{0}\right)^{2}}{H_{2}+G_{0} \eta_{2}} .
\end{aligned}
$$

To determine the effect of compressive axial load on the steady-state vibration amplitudes $A_{n 1}$ and $A_{n 2}$ of the system, the results under compressive axial load and those without axial load are compared. We introduce the relation

$$
\begin{gathered}
\varphi_{1}=\frac{A_{n 1}}{A_{n 1}^{0}}, \\
\varphi_{2}=\frac{A_{n 2}}{A_{n 2}^{0}} .
\end{gathered}
$$

We use nondimensional ratio

$$
s=\frac{F_{1}}{P},
$$

where $P=E I \pi^{2} / l^{2}$ is the smallest load at which the SWCNT ceases to be in stable equilibrium under axial compression. With the vibration mode number $n=3$ and the exciting frequency $\Omega=0.6 \omega_{n I I}$, the effects of compressive axial load on the steady-state vibration amplitudes $A_{n 1}$ and $A_{n 2}$ of the SWCNT are represented by the ratios $\varphi_{1}$ and $\varphi_{2}$, shown in Figures 4 and 5, respectively. As can be seen, the ratio $\varphi_{1}$ decreases with the increase of the axial compression, which implies that the magnitude of the steady-state vibration amplitude $A_{n 1}$ becomes smaller when the axial compression increases, and the ratio $\varphi_{2}$ increases with the increase of the axial compression, which implies that the magnitude of the steady-state vibration amplitude $A_{n 2}$ becomes larger when the axial compression increases.

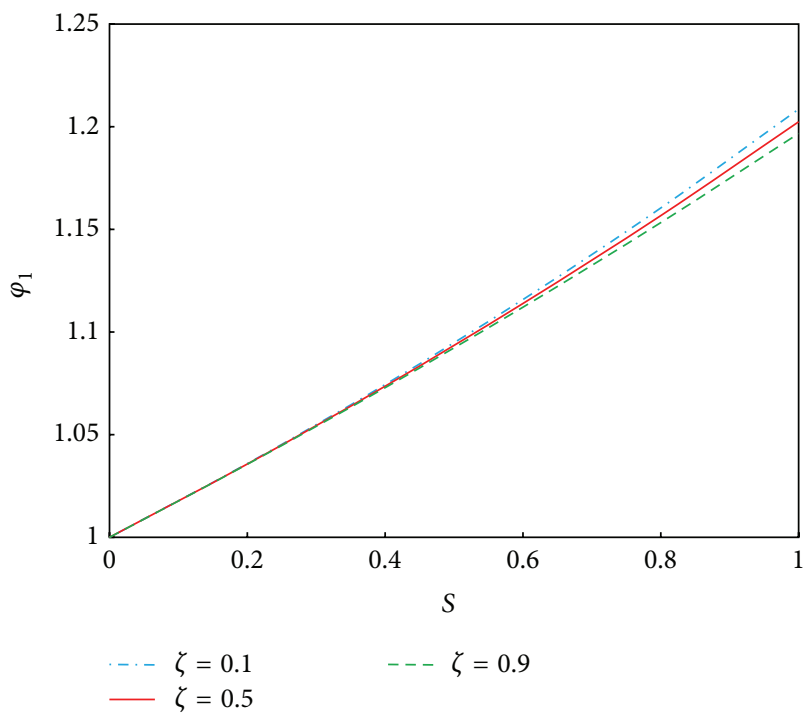

FIgURE 4: Relationship between ratio $\varphi_{1}$ and dimensionless parameter $s$ for different axial compression ratio $\zeta$.

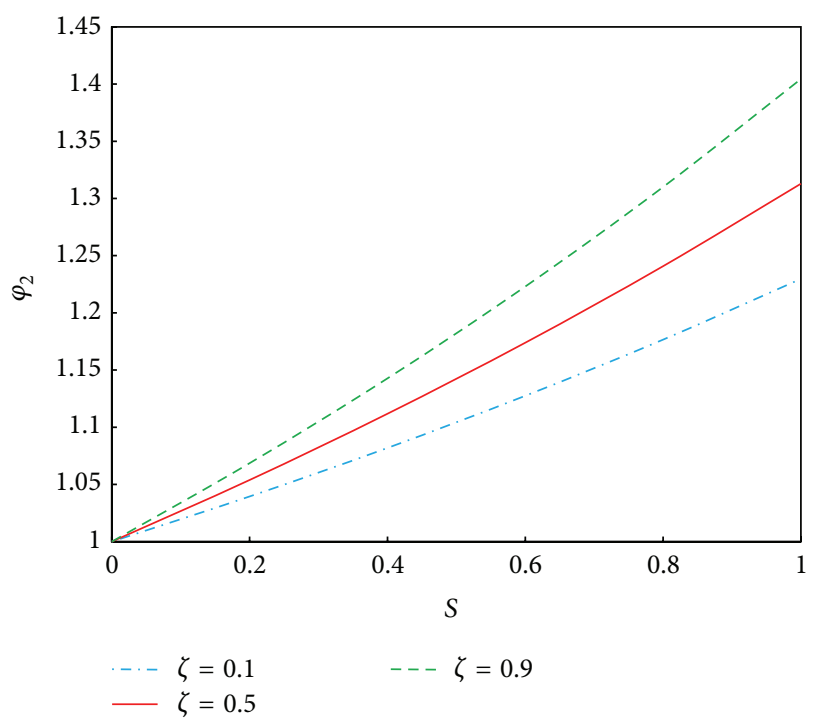

FIGURE 5: Relationship between ratio $\varphi_{2}$ and dimensionless parameter $s$ for different axial compression ratio $\zeta$.

Numerical values of the ratios $\varphi_{1}$ and $\varphi_{2}$ for different axial compression ratio $\zeta$ of the two SWCNTs are shown in Table 2. It can be observed that the effect of compressive axial load on the magnitude of $A_{n 1}$ is almost independent of the axial compression ratio $\zeta$ of the two SWCNTs whereas it is significantly dependent on the magnitude of $A_{n 2}$. 
TABLE 2: Steady-state vibration amplitudes ratios $\varphi_{1}$ and $\varphi_{2}$ of SWCNT for different compressive axial ratio $\zeta$.

\begin{tabular}{ccccccc}
\hline & & \multicolumn{3}{c}{$s$} \\
& 0 & 0.2 & 0.4 & 0.6 & 0.8 & 1 \\
\hline$\varphi_{1}(\zeta=0.1)$ & 1 & 1.0359 & 1.0744 & 1.1158 & 1.1604 & 1.2085 \\
\hline$\varphi_{1}(\zeta=0.5)$ & 1 & 1.0357 & 1.0736 & 1.1139 & 1.1567 & 1.2024 \\
\hline$\varphi_{1}(\zeta=0.9)$ & 1 & 1.0356 & 1.0729 & 1.1121 & 1.1533 & 1.1967 \\
\hline$\varphi_{2}(\zeta=0.1)$ & 1 & 1.0395 & 1.0819 & 1.1275 & 1.1766 & 1.2297 \\
\hline$\varphi_{2}(\zeta=0.5)$ & 1 & 1.0539 & 1.1118 & 1.1739 & 1.2408 & 1.3129 \\
\hline$\varphi_{2}(\zeta=0.9)$ & 1 & 1.0686 & 1.1427 & 1.2230 & 1.3100 & 1.4044 \\
\hline
\end{tabular}

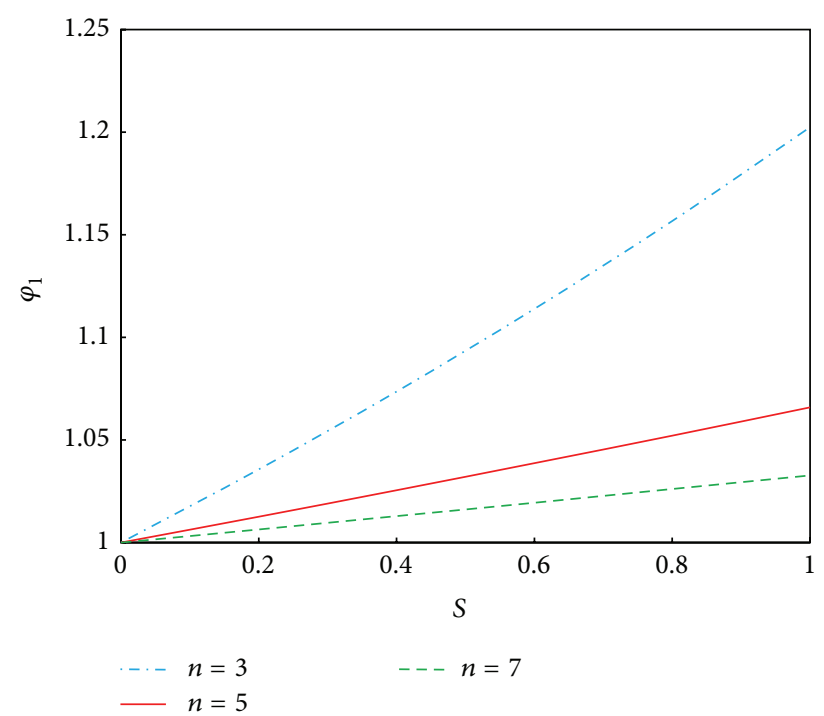

FIGURE 6: Relationship between ratio $\varphi_{1}$ and dimensionless parameter $s$ for different mode number $n$.

On the other hand, with the axial compression ratio $\zeta=0.5$ and the exciting frequency $\Omega=0.6 \omega_{n I I}$, steady-state vibration amplitudes $A_{n 1}$ and $A_{n 2}$ are shown in Figures 6 and 7 for different mode shape number $n$, respectively. It can be seen that, with the same axial compression, the ratios $\varphi_{1}$ and $\varphi_{2}$ diminish with the increasing vibration mode number $n$, which implies that the magnitudes of the steady-state vibration amplitudes $A_{n 1}$ and $A_{n 2}$ get smaller when the vibration mode number $n$ becomes larger.

Table 3 shows the effects of compressive axial load on the difference between the steady-state vibration amplitude ratios $\varphi_{1}$ and $\varphi_{2}$ of the SWCNT for different mode number $n$. It can be observed that the differences between ratios $\varphi_{1}$ and $\varphi_{2}$ of the SWCNT increase with increasing the dimensionless parameter $s$.

Table 4 shows the effects of length of SWCNT on the difference between the steady-state vibration amplitude ratios $\varphi_{1}$ and $\varphi_{2}$ of the SWCNT for mode number $n=5$ and axial compression ratio $\zeta=0.5$. It can be observed that the differences between ratios $\varphi_{1}$ and $\varphi_{2}$ of the SWCNT decrease with increasing of length of SWCNT.

We accounted the results for three cases of shear foundation modulus of Pasternak layer $G_{0}=0,100,1000$. With the vibration mode number $n=3$ and the exciting frequency

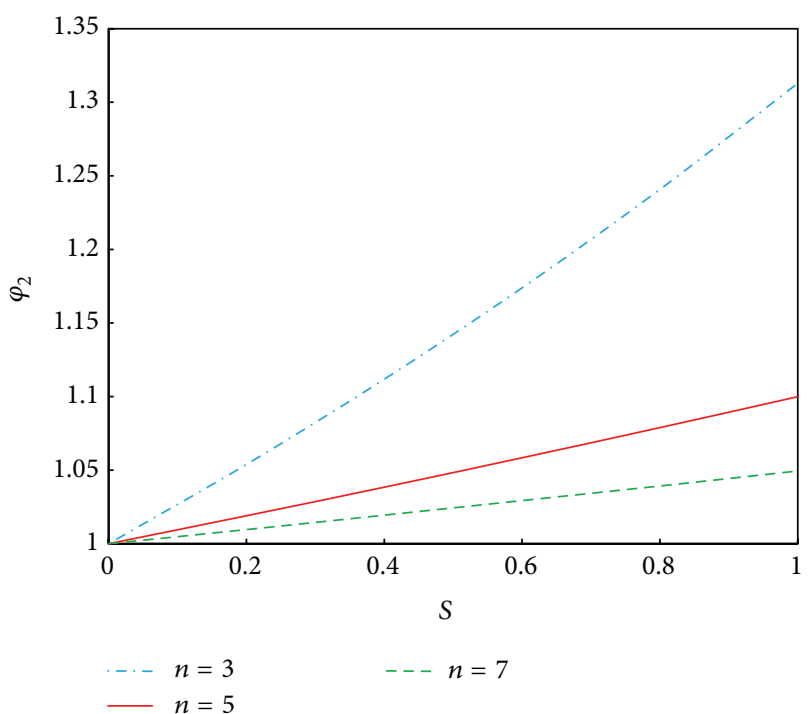

FIGURE 7: Relationship between ratio $\varphi_{2}$ and dimensionless parameter $s$ for different mode number $n$.

TABLE 3: Steady-state vibration amplitudes ratios $\varphi_{1}$ and $\varphi_{2}$ for different mode shape number $n$.

\begin{tabular}{ccccccc}
\hline & & \multicolumn{3}{l}{$s$} \\
& 0 & 0.2 & 0.4 & 0.6 & 0.8 & 1 \\
\hline$\varphi_{1}(n=3)$ & 1 & 1.0357 & 1.0736 & 1.1139 & 1.1567 & 1.2024 \\
\hline$\varphi_{1}(n=5)$ & 1 & 1.0126 & 1.0255 & 1.0387 & 1.0521 & 1.0659 \\
\hline$\varphi_{1}(n=7)$ & 1 & 1.0064 & 1.0129 & 1.0194 & 1.0261 & 1.0327 \\
\hline$\varphi_{2}(n=3)$ & 1 & 1.0539 & 1.1118 & 1.1739 & 1.2408 & 1.3129 \\
\hline$\varphi_{2}(n=5)$ & 1 & 1.0190 & 1.0384 & 1.0584 & 1.0789 & 1.0999 \\
\hline$\varphi_{2}(n=7)$ & 1 & 1.0096 & 1.0194 & 1.0293 & 1.0392 & 1.0494 \\
\hline
\end{tabular}

TABLE 4: Steady-state vibration amplitudes ratios $\varphi_{1}$ and $\varphi_{2}$ for different length of SWCNT, $l$.

\begin{tabular}{lcccccc}
\hline & & \multicolumn{5}{c}{$s$} \\
& 0 & 0.2 & 0.4 & 0.6 & 0.8 & 1 \\
\hline$\varphi_{1}\left(l_{1}=l\right)$ & 1 & 1.0126 & 1.0255 & 1.0387 & 1.0521 & 1.0659 \\
\hline$\varphi_{1}\left(l_{1}=5 l\right)$ & 1 & 1.0126 & 1.0255 & 1.0386 & 1.0521 & 1.0658 \\
\hline$\varphi_{1}\left(l_{1}=10 l\right)$ & 1 & 1.0125 & 1.0252 & 1.0382 & 1.0515 & 1.0650 \\
\hline$\varphi_{2}\left(l_{1}=l\right)$ & 1 & 1.0190 & 1.0384 & 1.0584 & 1.0789 & 1.0999 \\
\hline$\varphi_{2}\left(l_{1}=5 l\right)$ & 1 & 1.0190 & 1.0384 & 1.0583 & 1.0787 & 1.0997 \\
\hline$\varphi_{2}\left(l_{1}=10 l\right)$ & 1 & 1.0188 & 1.0380 & 1.0577 & 1.0778 & 1.0984 \\
\hline
\end{tabular}

$\Omega=0.6 \omega_{n I I}$, the effects of Pasternak layer on the steady-state vibration amplitudes $A_{n 1}$ and $A_{n 2}$ of the Rayleigh beam are represented by the ratios $\varphi_{1}$ and $\varphi_{2}$, shown in Table 5 .

Numerical values of the ratios $\varphi_{1}$ and $\varphi_{2}$ for different shear foundation modulus of Pasternak layer with different axial compression ratio $\zeta$ of the two beams are shown in Table 5. Numerical results of the ratios $\varphi_{1}$ and $\varphi_{2}$ show that the difference with ratios $\varphi_{1}$ and $\varphi_{2}$ decreases with the increase of shear modulus of the Pasternak layer for different axial compression ratio $\zeta$, but it can be observed that the effect of shear foundation modulus of Pasternak 
TABLE 5: Effects of shear foundation modulus of Pasternak layer on the steady-state vibration amplitudes ratios $\varphi_{1}$ and $\varphi_{2}$ for different compressive axial ratio $\zeta$.

\begin{tabular}{|c|c|c|c|c|c|c|}
\hline & \multicolumn{6}{|c|}{$s$} \\
\hline & 0 & 0.2 & 0.4 & 0.6 & 0.8 & 1 \\
\hline \multicolumn{7}{|l|}{$\varphi_{1}(\zeta=0.1)$} \\
\hline$\left(G_{0}=0\right)$ & 1 & 1.0351 & 1.0723 & 1.112 & 1.1544 & 1.1999 \\
\hline$\left(G_{0}=100\right)$ & 0.9999 & 1.035 & 1.0723 & 1.1119 & 1.1543 & 1.1998 \\
\hline$\left(G_{0}=1000\right)$ & 0.9993 & 1.0343 & 1.0715 & 1.111 & 1.1533 & 1.1987 \\
\hline \multicolumn{7}{|l|}{$\varphi_{1}(\zeta=0.5)$} \\
\hline$\left(G_{0}=0\right)$ & 1 & 1.0351 & 1.0722 & 1.1112 & 1.1526 & 1.1965 \\
\hline$\left(G_{0}=100\right)$ & 0.9999 & 1.0351 & 1.0721 & 1.1111 & 1.1525 & 1.1963 \\
\hline$\left(G_{0}=1000\right)$ & 0.9993 & 1.0343 & 1.0713 & 1.1102 & 1.1515 & 1.1952 \\
\hline \multicolumn{7}{|l|}{$\varphi_{1}(\zeta=0.9)$} \\
\hline$\left(G_{0}=0\right)$ & 1 & 1.0352 & 1.072 & 1.1107 & 1.1513 & 1.194 \\
\hline$\left(G_{0}=100\right)$ & 0.9999 & 1.0351 & 1.072 & 1.1106 & 1.1512 & 1.1938 \\
\hline$\left(G_{0}=1000\right)$ & 0.9993 & 1.0344 & 1.0711 & 1.1097 & 1.1501 & 1.1927 \\
\hline \multicolumn{7}{|l|}{$\varphi_{2}(\zeta=0.1)$} \\
\hline$\left(G_{0}=0\right)$ & 1 & 1.0386 & 1.0796 & 1.1232 & 1.1699 & 1.22 \\
\hline$\left(G_{0}=100\right)$ & 0.9925 & 1.0308 & 1.0714 & 1.1147 & 1.1611 & 1.2108 \\
\hline$\left(G_{0}=1000\right)$ & 0.9255 & 0.9611 & 0.999 & 1.0393 & 1.0824 & 1.1287 \\
\hline \multicolumn{7}{|l|}{$\varphi_{2}(\zeta=0.5)$} \\
\hline$\left(G_{0}=0\right)$ & 1 & 1.0528 & 1.109 & 1.169 & 1.2332 & 1.302 \\
\hline$\left(G_{0}=100\right)$ & 0.9925 & 1.0448 & 1.1006 & 1.1602 & 1.2238 & 1.2921 \\
\hline$\left(G_{0}=1000\right)$ & 0.9255 & 0.9742 & 1.0261 & 1.0816 & 1.1408 & 1.2044 \\
\hline \multicolumn{7}{|l|}{$\varphi_{2}(\zeta=0.9)$} \\
\hline$\left(G_{0}=0\right)$ & 1 & 1.0673 & 1.1398 & 1.2181 & 1.3028 & 1.3944 \\
\hline$\left(G_{0}=100\right)$ & 0.9925 & 1.0592 & 1.1312 & 1.2089 & 1.2929 & 1.3839 \\
\hline$\left(G_{0}=1000\right)$ & 0.9255 & 0.9876 & 1.0546 & 1.1269 & 1.2051 & 1.2897 \\
\hline
\end{tabular}

layer on the magnitude of $A_{n 1}$ is almost independent of the axial compression ratio $\zeta$ of the two beams whereas it is significantly dependent on the magnitude of $A_{n 2}$.

\section{Conclusions}

Based on the Rayleigh beam theory, the forced transverse vibrations of a closed double single-walled carbon nanotube (SWCNT) system containing a fluid with a Pasternak layer in-between, under compressive axial loading for one case of particular excitation loading, are studied. The dynamic responses of the system caused by arbitrarily distributed continuous loads are obtained. The effect of compressive axial load and length of SWCNT on the forced vibrations of the double single-walled carbon nanotube system is discussed for one case of particular excitation loading. The magnitudes of the steady-state vibration amplitudes of the beam are dependent on the axial compression and length of SWCNT. The effect of compressive axial load on the magnitude of $A_{n 1}$ is almost independent of the axial compression ratio $\zeta$ of the two SWCNTs whereas it is significantly dependent on the magnitude of $A_{n 2}$. Also, it is shown with the same axial compression that the ratios $\varphi_{1}$ and $\varphi_{2}$ diminish with the increasing vibration mode number $n$, which implies that the magnitudes of the steady-state vibration amplitudes $A_{n 1}$ and $A_{n 2}$ get smaller when the vibration mode number $n$ becomes larger. The ratio $\varphi_{1}$ decreases with the increase of the axial compression, which implies that the magnitude of the steady-state vibration amplitude $A_{n 1}$ becomes smaller when the axial compression increases, and the ratio $\varphi_{2}$ increases with the increase of the axial compression, which implies that the magnitude of the steady-state vibration amplitude $A_{n 2}$ becomes larger when the axial compression increases. The differences between ratios $\varphi_{1}$ and $\varphi_{2}$ of the SWCNT decrease with increasing of length of SWCNT. The SWCNT-type dynamic absorber is a new concept of a dynamic vibration absorber (DVA), which can be applied to suppress excessive vibrations of corresponding SWCNT systems. At a given vibration amplitude, increase of the nanotube length leads to decrease of the frequencies. Also we concluded that the ratios $\varphi_{1}$ and $\varphi_{2}$ decrease with increasing of the shear foundation modulus of Pasternak layer $\left(G_{0}\right)$.

\section{Nomenclature}

$w=w(x, t): \quad$ Transverse displacements of the carbon

nanotubes

$\partial w / \partial x: \quad$ Global rotation of the cross section

$T_{n i}(t): \quad$ Unknown time function

$X_{n}(x)$ : Known mode shape function for simply supported carbon nanotube

$S_{n i}(t)(i=I, I I)$ : Unknown time function corresponding

$\delta_{m n}: \quad$ Kronecker delta function

$X_{m}: \quad$ Eigenfunction

$q(x): \quad$ Amplitude of the load

$\Omega: \quad \quad$ Exciting frequency of the load

$A_{n 1}^{0}, A_{n 2}^{0}$ : Steady-state vibration amplitudes of the two carbon nanotubes without axial compression

$\omega_{n}: \quad$ Natural frequencies of the system

A: $\quad$ Cross section area of the hollow

cylindrical nanotube

E: $\quad$ Young's modulus

I: $\quad$ Second moment of inertia of the nanotube

$\rho_{C}: \quad$ Mass density of the carbon nanotube

$\rho_{f}: \quad$ Mass density of the fluid

$K$ : Winkler foundation modulus

$G_{0}: \quad$ Shear foundation modulus

$d: \quad$ Inner diameter

$t_{C}: \quad$ Thickness.

\section{Conflict of Interests}

The authors declare that there is no conflict of interests regarding the publication of this paper.

\section{References}

[1] E. W. Wong, P. E. Sheehan, and C. M. Lieber, "Nanobeam mechanics: elasticity, strength, and toughness of nanorods and nanotubes," Science, vol. 277, no. 5334, pp. 1971-1975, 1997. 
[2] P. Poncharal, Z. L. Wang, D. Ugarte, and W. A. Heer, "Electrostatic deflections and electromechanical resonances of carbon nanotubes," Science, vol. 283, no. 5407, pp. 1513-1516, 1999.

[3] J. Yoon, C. Q. Ru, and A. Mioduchowski, "Noncoaxial resonance of an isolated multiwall carbon nanotube," Physical Review BCondensed Matter and Materials Physics, vol. 66, Article ID 233402, 2002.

[4] C. Li and T.-W. Chou, "Vibrational behaviors of multiwalledcarbon-nanotube-based nanomechanical resonators," Applied Physics Letters, vol. 84, pp. 121-123, 2004.

[5] C. Q. Ru, "Elastic models for carbon nanotubes," Encyclopedia of Nanoscience and Nanotechnology, vol. 2, pp. 731-744, 2004.

[6] L. F. Wang and H. Y. Hu, "Flexural wave propagation in singlewalled carbon nanotubes," Physical Review B, vol. 71, no. 19, Article ID 195412, 2005.

[7] Y. Q. Zhang, G. R. Liu, and X. Y. Xie, "Free transverse vibrations of double-walled carbon nanotubes using a theory of nonlocal elasticity," Physical Review B, vol. 71, Article ID 195404, pp. 195404, 2005.

[8] C. M. Wang, V. B. C. Tan, and Y. Y. Zhang, "Timoshenko beam model for vibration analysis of multi-walled carbon nanotubes," Journal of Sound and Vibration, vol. 294, no. 4, pp. 1060-1072, 2006.

[9] W.-J. Chang and H.-L. Lee, "Free vibration of a single-walled carbon nanotube containing a fluid flow using the Timoshenko beam model," Physics Letters A, vol. 373, no. 10, pp. 982-985, 2009.

[10] J. M. Seelig and W. H. Hoppmann, "Impact on an elastically connected double-beam system," Journal of Applied Mechanics, vol. 31, no. 4, p. 621, 1964.

[11] Z. Oniszczuk, "Free transverse vibrations of elastically connected simply supported double-beam complex system," Journal of Sound and Vibration, vol. 232, no. 2, pp. 387-403, 2000.

[12] Z. Oniszczuk, "Forced transverse vibrations of an elastically connected complex simply supported double-beam system," Journal of Sound and Vibration, vol. 264, no. 2, pp. 273-286, 2003.

[13] Y. Q. Zhang, Y. Lu, S. L. Wang, and X. Liu, "Vibration and buckling of a double-beam system under compressive axial loading," Journal of Sound and Vibration, vol. 318, no. 1-2, pp. 341-352, 2008.

[14] Y. Q. Zhang, Y. Lu, and G. W. Ma, "Effect of compressive axial load on forced transverse vibrations of a double-beam system," International Journal of Mechanical Sciences, vol. 50, no. 2, pp. 299-305, 2008.

[15] V. Stojanović, P. Kozić, R. Pavlović, and G. Janevski, "Effect of rotary inertia and shear on vibration and buckling of a double beam system under compressive axial loading," Archive of Applied Mechanics, vol. 81, no. 12, pp. 1993-2005, 2011.

[16] V. Stojanović and P. Kozić, "Forced transverse vibration of Rayleigh and Timoshenko double-beam system with effect of compressive axial load," International Journal of Mechanical Sciences, vol. 60, no. 1, pp. 59-71, 2012.

[17] V. Stojanović, P. Kozić, and G. Janevski, "Buckling instabilities of elastically connected timoshenko beams on an elastic layer subjected to axial forces," Journal of Mechanics of Materials and Structures, vol. 7, no. 4, pp. 363-374, 2012. 

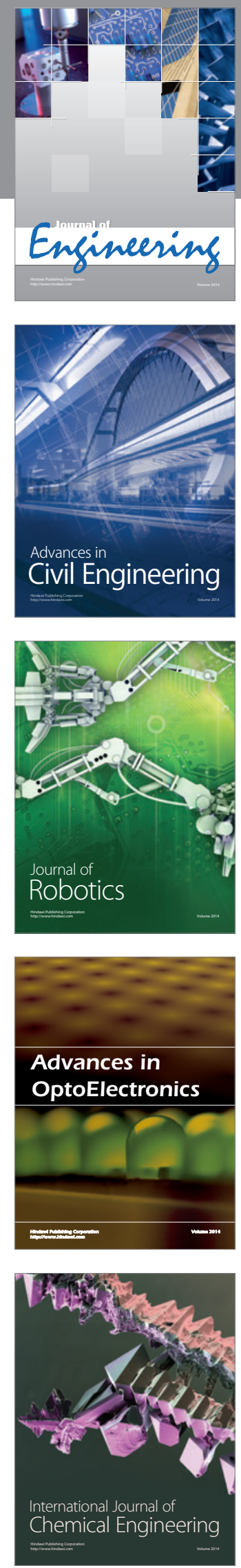

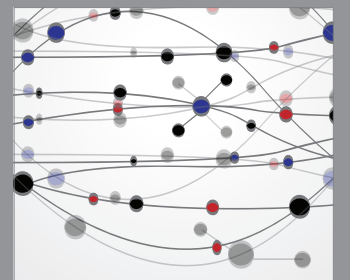

The Scientific World Journal
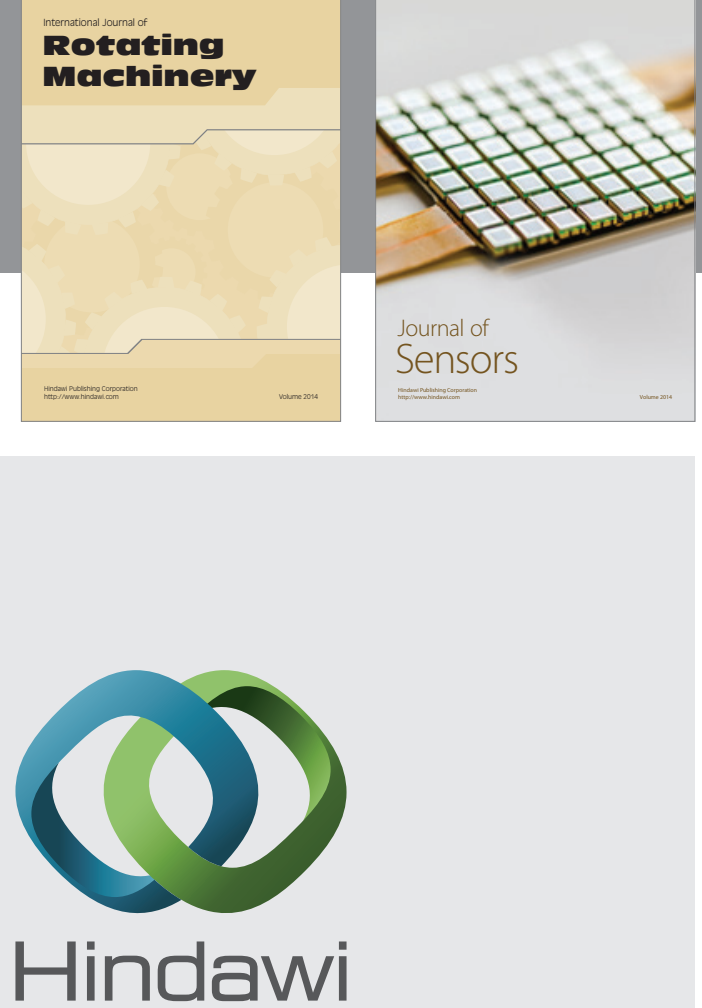

Submit your manuscripts at http://www.hindawi.com
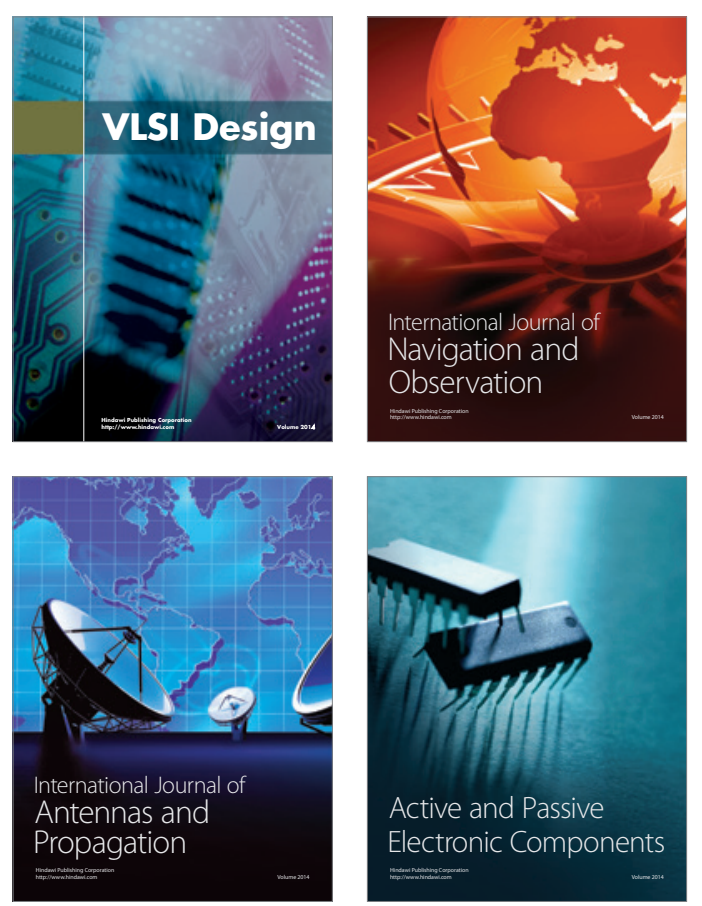
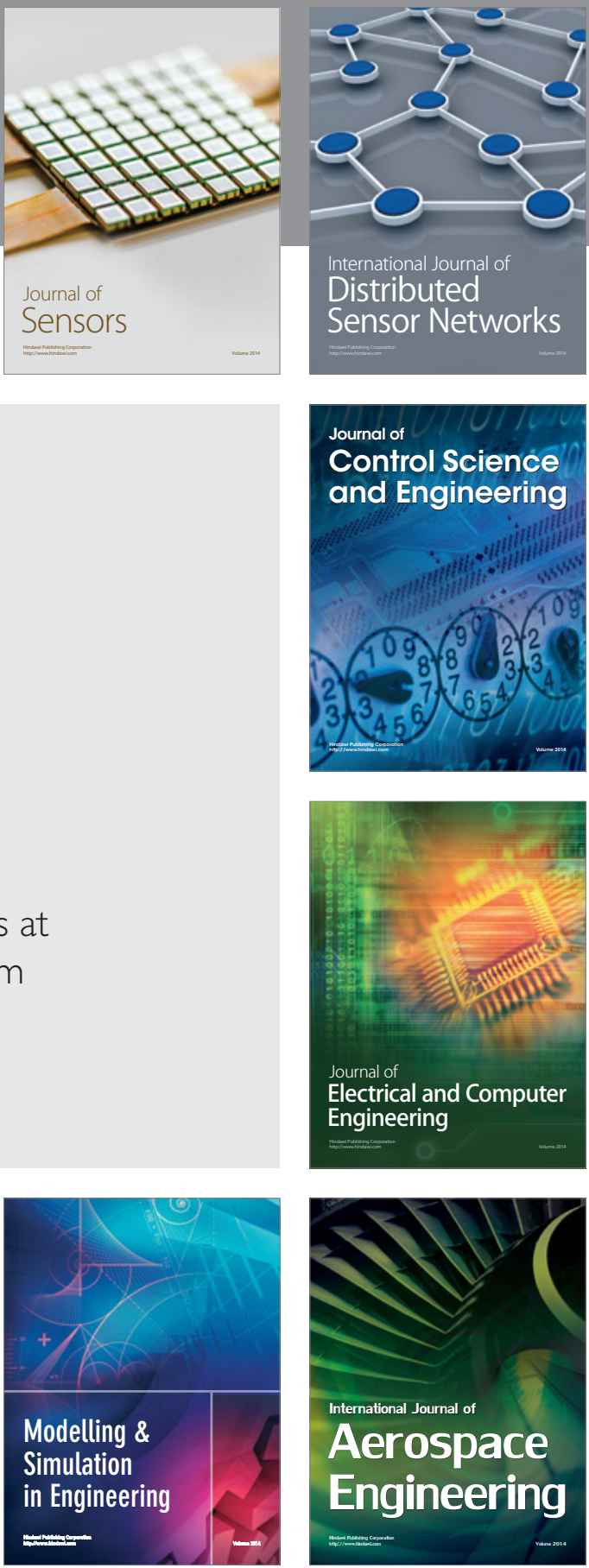

Journal of

Control Science

and Engineering
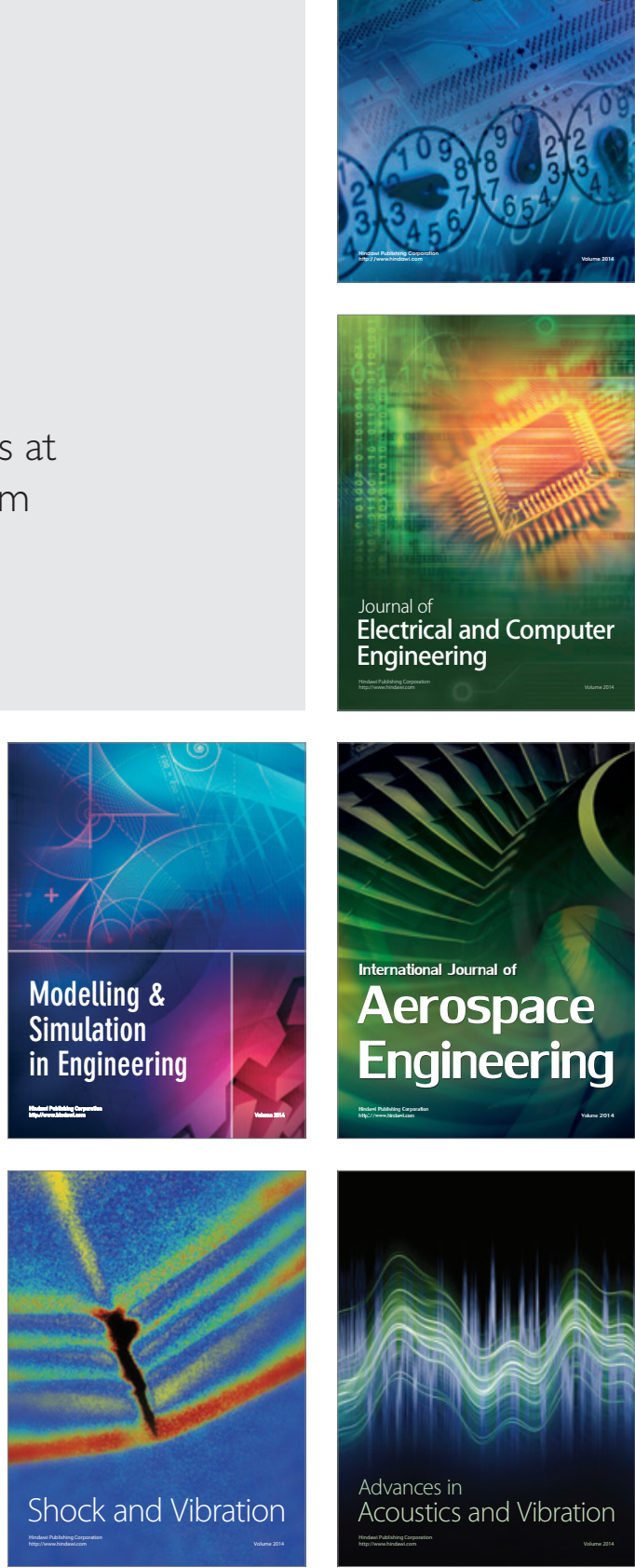